\title{
Short- and Long-Term Outcome of Chronic Dialyzed Patients Admitted to the ICU and Assessment of Prognosis Factors: Results of a 6-Year Cohort Study
}

\author{
Rémi Trusson, $\mathrm{MD}^{1}$; Vincent Brunot, $\mathrm{MD}^{2}$; Romaric Larcher, $\mathrm{MD}^{2,3}$; Laura Platon, $\mathrm{MD}^{2}$; \\ Noémie Besnard, $\mathrm{MD}^{2}$; Olivier Moranne, $\mathrm{MD}, \mathrm{PhD}^{4,5}$; Saber Barbar, $\mathrm{MD}, \mathrm{PhD}^{1}$; \\ Jean-Emmanuel Serre, $\mathrm{MD}^{6}$; Kada Klouche, $\mathrm{MD}, \mathrm{PhD}^{2,3}$
}

\begin{abstract}
Ohjectives: Data about end-stage kidney disease patients admitted to the ICU are scarce, dated, and mostly limited to short-term survival. The aim of this study was to assess the short- and longterm outcome and to determine the prognostic factors for endstage kidney disease patients admitted to the ICU.

Design: Prospective observational study.
\end{abstract}

Setting: Medical ICUs in two university hospitals.

Patients: Consecutive end-stage kidney disease patients admitted in two ICUs between 2012 and 2017.

Intervention: None.

Measurements and Main Results: Renal replacement therapy variables, demographic, clinical, and biological data were collected. The requirement of mechanical ventilation and vasopressive drugs were also collected. In-ICU and one-year mortality were estimated and all data were analyzed in order to identify predictive factors of short and long-term mortality. A total of 140 patients were included, representing $1.7 \%$ of total admissions over the study period. Septic shock was the main reason for admission mostly of pulmonary origin. Median Simplified Acute Physiology Score II and Sequential Organ Failure Assessment score were at 63 and 6.7 respectively. In-ICU, hospital, and 1-year mortality were $41.4 \%, 46.4 \%$, and $63 \%$, respectively. ICU mortality was significantly higher as compared with ICU control group non-end-

'Department of Intensive Care Medicine, University Hospital, Nimes, France.

${ }^{2}$ Department of Intensive Care Medicine, Lapeyronie University Hospital, Montpellier, France.

${ }^{3}$ PhyMedExp, University of Montpellier, INSERM, CNRS, Montpellier, France.

${ }^{4}$ Nephrology-Dialysis-Apheresis Unit, University Hospital, Nimes, France.

5UPRES EA2415, Laboratory of Biostatistics, Epidemiology, Clinical Research and Health Economics, University of Montpellier, Montpellier, France.

${ }^{6}$ Department of Nephrology, Lapeyronie University Hospital, Montpellier, France. stage kidney disease ( $25 \%$ vs $41.4 \% ; p=0.005$ ). By multivariate analysis, the short-term outcome was significantly associated with nonrenal Sequential Organ Failure Assessment score, and with the requirement of mechanical ventilation or/and vasoconstrictive agents during ICU stay. One-year mortality was associated with increased dialysis duration $(>3 \mathrm{yr})$ and phosphatemia $(>2.5 \mathrm{mmol} / \mathrm{L})$, with lower albuminemia $(<30 \mathrm{~g} / \mathrm{L})$ and nonrenal Sequential Organ Failure Assessment greater than 8.

Conclusions: End-stage kidney disease patients presented frequently severe complications requiring critical care that induced significant short- and long-term mortality. ICU and hospital mortality depended mainly on the severity of the critical event reflected by Sequential Organ Failure Assessment score and the need of mechanical ventilation and/or catecholamines. One-year mortality was associated with both albuminemia and phosphatemia and with prior duration of chronic dialysis treatment, and with organ failure at ICU admission. (Crit Care Med 2020; XX:00-00) Key Words: chronic dialysis; chronic renal disease; end-stage renal disease; intensive care unit; outcome; prognosis

1 The prevalence of end-stage kidney disease (ESKD) is rising steadily worldwide (1-5). The majority of these patients will undergo either chronic hemodialysis or peritoneal dialysis (2). In France, the incidence of ESKD patients requiring chronic dialysis is about 164 per million population and is increasing of $1 \%$ per year $(6,7)$. Dialysis patients are particularly susceptible to infections due to uremia-related immune deficiency, older age, and comorbidities including diabetes mellitus and arterial hypertension. They have also a higher risk of adverse cardiac events including myocardial ischemia and sudden cardiac death (8). Consequently, dialysis patients frequently experience severe and life-threatening complications that require critical care (9-14).

ESKD patients are frequently admitted to the ICU much more than the general population $(10,15-17)$. They bear a poor prognosis with a substantial risk of morbidity and mortality 
(10, 11, 18-23). Aggressive life support has been sometimes considered questionable or even futile. Several studies aimed to identify chronic renal disease (CRD) patients susceptible to benefit from critical care by the research of mortality risk factors. Indeed, an elevated phosphatemia and multiple organ failure at admission were found to be associated with mortality $(9-11,24,25)$. Critical care severity scores were also correlated with the mortality but overestimated it probably due to the taken into account of abnormal variables related to ESKD $(9,10,16,26,27)$. In these recent years, substantial advances in management of both dialysis and critical care have been realized and may significantly modify the prognosis of such patients. These better provisions of care may be associated with improved mortality rates. Previous investigations were mostly performed and published more than 10 years ago $(9,10,25)$. Furthermore, they mainly investigate only in-ICU mortality or short-term survival. This lack of recent comprehensive data prompted us therefore to carry out a 6-year analysis of a large cohort of CRD patients admitted to an ICU in order to study epidemiology and the clinical features of severe complications and to reassess the factors of prognosis and short- and longterm mortality.

\section{PATIENTS AND METHODS}

This observational prospective study was performed in two medical ICUs of university hospitals in Montpellier and Nîmes, France. The Institutional Review Board approved the study and waived the need for informed consent. In both hospitals, chronic dialyzed (CD) patients are followed by nephrologists and admitted in Nephrology and Transplantation Department for any complication. Whenever any of these patients present a severe and life-threatening condition necessitating prolonged mechanical ventilation (MV) or/and hemodynamic support, they are transferred to ICUs and followed simultaneously with the dialysis team. Our policy is that the decision to admit to ICU and to intensively treat this population should be made by both the intensivists and the nephrologists and according to the patient and family wishes.

\section{Study Population}

All consecutive CD patients admitted to both ICUs from January 2012 to December 2017 were included in the study. A patient was considered CD if he or she was undergoing hemodialysis or peritoneal dialysis for at least 3 months. ESKD patients admitted to the ICU after scheduled surgery without postoperative complications, trauma or attempted suicide, or with a Simplified Acute Physiology Score II (SAPS II) less than 15 were excluded. When a patient was admitted twice or more, only the first ICU admission was considered.

During ICU stay, the choice of renal replacement therapy (RRT) modality was depending on patient hemodynamic stability and was daily reevaluated. Patients with hemodynamic instability or severe fluid overload were preferentially treated with continuous venovenous hemodia/filtration (CVVHD/F) or sustained low-efficiency daily dialysis filtration (SLEDD-f) and with intermittent hemodialysis when they had or recovered hemodynamic stability. Patients previously chronically treated by peritoneal dialysis were also switched to hemodialysis/filtration during their ICU stay.

\section{Data Collected}

Demographic, clinical, and biological data were collected prospectively and included age, sex, comorbidities, and Charlson Comorbidity Index (28). The following variables were also collected: primary renal disease, type and duration of dialysis, vascular access, previous renal transplantation, mean albuminemia level in the last 3 months prior to the acute episode. The type, medical or surgical, and reason of ICU admission were noted. An admission was considered as surgical when a surgery was performed 1 week before or after it; surgery was considered urgent if performed within 24 hours of its indication. Time elapsed from hospital to ICU admission was recorded and differentiated into direct ICU admission $(0 \mathrm{~d})$, less or more than 7 days. Upon ICU admission, clinical and biological variables including albuminemia, lactatemia, serum bicarbonate, and phosphoremia, were monitored. The severity of the disease was assessed 24 hours after admission using the SAPS II (26) and the Sequential Organ Failure Assessment (SOFA) scores excluding renal failure (29). During the ICU stay, the need for and the duration of invasive MV, and the use of vasoactive drugs were monitored. During ICU stay, we also assessed the occurrence of complications including ventilator-acquired pneumonia, catheter-related infection, and hemorrhagic or thrombotic events.

\section{Study Outcomes}

ICU and hospital length of stays and outcome including ICU, in-hospital, 90 days, and 1-year mortality were also recorded. The survival state and date of death, if applicable, were collected using computerized medical file records or by phone contact with dialysis center medical referent of each patient. Death by limitation of care, usually stopping RRT, was informed. We compared survivors and nonsurvivors for all the above variables in order to identify the predictive factors of ICU and 1-year mortality.

The studied population was compared with a matched control group (by age, sex, SOFA and SAPS II scores, and main ICU management) in term of ICU mortality.

\section{Statistical Analysis}

Qualitative data were described as numbers and percentages, and quantitative data as means with sDs, except for non-Gaussian variables which were displayed as medians with 25 th and 75th percentiles. ICU survival was defined as the time between ICU admission and ICU discharge, and in-hospital survival as the time between ICU admission and hospital discharge. Patients were classified as survivors if they were discharged, respectively, from the ICU and from the hospital. Because hospital death and discharge are competing risks, survival was analyzed using cumulative incidence estimates. One-year survival was defined 1 year starting from ICU admission. Data of patients who died afterward were censored at 3 months, 6 
months, and 1-year follow-up, respectively. One-year survival was estimated using the Kaplan-Meier method. ICU mortality of this population was compared to an ICU non-ESKD control group that was matched by age, sex, SOFA score, and ICU management.

The analysis of ICU prognostic factors used a cause-specific Cox model, in the context of competing risks. The analysis of 1-year survival prognostic factors also involved a standard Cox regression. Factors associated with survival in the univariate analysis (cutoff of $p \leq 0.1$ ) were included in the multivariate analysis, and a step-by-step variable selection procedure was performed. Hazard ratios were given with a 95\% CI.

All tests were two-tailed with a statistical significance level of $5 \%$. All statistical analyses were performed using SPSS 18.0 software (IBM, Armonk, NY) and GraphPad Prismversion 5.00 (GraphPad Software, San Diego, CA).

\section{RESULTS}

\section{Population}

During the study period, 8,235 patients were admitted to ICUs. One-hundred forty of these patients required chronic renal dialysis and entered the study representing $1.7 \%$ of all ICU admissions (Fig. S1, Supplemental Digital Content 1, http:// links.lww.com/CCM/F524; legend, Supplemental Digital Content 6, http://links.lww.com/CCM/F529). Also, the studied population represented $1.4 \%$ of ESKD patients $(140 / 1,802)$ who had emergency hospital admission.

Demographic data, comorbidities, and characteristics of chronic renal failure of the studied population are displayed in Table 1. Ninety-four males and 46 females (sex ratio male/ female: 2.04$)$ with median age at 70 years $(62-76 \mathrm{yr})$ were studied. More than $80 \%$ of the patients $(113 / 140)$ were over 60 years old at admission. Most of the patients had comorbidities mainly cardiopathy $(82 / 140,58.6 \%)$, dyslipidemia (77/140, $55 \%)$, peripheral arterial disease $(73 / 140,52.1 \%)$, and diabetes mellitus (62/140, 44.3\%). Median Charlson Comorbidity Index was 6 (4.5-7). CRD was more frequently hypertensioninduced nephropathy (34.3\%), and diabetic nephropathy (24.3\%). Median time dialysis duration before admission was 2.5 years $(0.7-6 \mathrm{yr})$, one of three of patients being dialyzed for more than 5 years and one of three for less than 1 year. Patients were treated mostly by hemodialysis (123/140, 88\%), including 94 on arteriovenous fistula and 29 on tunneled twin central venous catheter, and the remaining 17 patients by peritoneal dialysis. Eighteen patients benefited previously from a kidney transplant. Previous albuminemia was less than $35 \mathrm{~g} / \mathrm{L}$ in 78 patients (56\%).

Reasons of admission were mostly medical (105/140, 75\%). Of note, surgery was performed urgently in 29 patients $(21 \%)$. Near half of the patients were admitted directly, whereas $35 \%$ of them were hospitalized 7 days or less before ICU admission. Septic shock was the most frequent cause of ICU admission (33\%), mainly of pulmonary origin. Other causes for admission included mainly a cardiovascular event (myocardial infarction, mesenteric ischemia, stroke: $42 / 140,30 \%)$ and hemorrhagic shock (20/140,

TABLE 1. Demographic and Characteristics of the Studied Population

Baseline Characteristics

Age, $y r$, mean $\pm \mathrm{sD}$

Sex ratio male/female

Comorbidities

Charlson Comorbidity Index, mean \pm SD

Ischemic heart disease and/or congestive heart failure, $n(\%)$

Ischemic heart disease, $n(\%)$

Congestive heart failure, $n(\%)$

Dyslipidemia, $n$ (\%)

Peripheral arterial disease, $n(\%)$

Diabetes mellitus, $n(\%)$

Chronic obstructive pulmonary disease, $n(\%)$

Cerebrovascular disease, $n(\%)$

Hepatic cirrhosis child B or C, $n(\%)$

Chronic renal disease, $n(\%)$

Hypertension-induced nephropathy

Diabetic nephropathy

Glomerulonephritis or systemic disease

Chronic interstitial nephritis

Polycystic kidney disease

Other disease or undetermined

Prior renal transplant, $n$ (\%)

Prior dialysis duration, yr, median

(interquartile range 25-75)

Less than $1 \mathrm{yr}, n(\%)$

Between 1 and $3 \mathrm{yr}, n$ (\%)

Between 3 and $5 \mathrm{yr}, n$ (\%)

More than $5 \mathrm{yr}, n(\%)$

Unknown or not available, $n(\%)$

Dialysis modality, $n(\%)$

Hemodialysis

Arteriovenous fistula, vascular prosthesis, or bridging

Tunneled central venous catheters

Peritoneal dialysis

Albuminemia $<35 \mathrm{~g} / \mathrm{L}$

14.3\%). These results are summarized in Table S1 (Supplemental Digital Content 2, http://links.lww.com/CCM/F525). Twenty-six patients $(26 / 140,18.6 \%)$ presented a cardio-circulatory arrest prior to ICU admission. $n=140$

$68.5 \pm 12.3$

94/46:2.04

$5.8 \pm 2.1$

$82(58.6)$

$44(31.4)$

$61(43.6)$

$77(55)$

$73(52.1)$

$62(44.3)$

$23(20)$

$23(16.4)$

$3(2.6)$

48 (34.3)

34 (24.3)

27 (19.3)

$12(8.6)$

$5(3.6)$

$14(10)$

18 (13)

$2.38(0.74-6.07)$

48 (34.3)

25 (17.9)

21 (15)

44 (31.4)

$2(1.4)$

123 (88)

$94(67.1)$

32 (22.9)

$52(56.5)$ 
At admission, high mean SAPS II $(63 \pm 22)$ and nonrenal SOFA scores $(6.7 \pm 3.8)$ underlined the severity of the patient illnesses (Table 2). Mean phosphatemia and albuminemia were $1.65 \pm 0.86 \mathrm{mmol} / \mathrm{L}$ and $27.3 \pm 7.7 \mathrm{~g} / \mathrm{L}$, respectively. Eightyeight patients $(63 \%)$ had serum albumin of less than $30 \mathrm{~g} / \mathrm{L}$ and 48 patients $(34 \%)$ had a lactatemia greater than $4 \mathrm{mmol} / \mathrm{L}$ (Table 2).

During the ICU stay, 100 patients (71.4\%) received invasive MV; $94(67.1 \%)$ received vasoconstrictive agents for a median duration of 2 days ( $0-4.5 \mathrm{~d})$ and 2 days $(0-4 \mathrm{~d})$, respectively (Table 2). In the first ICU days, RRT modality was CVVHD/F or SLEDD-f and was changed to intermittend hemodialysis/filtration when hemodynamic stability was obtained. Permanent vascular access was never used. Vascular access consisted of a double-lumen central venous dialysis catheter of $15.5 \mathrm{~F}$ caliber placed at right internal jugular or femoral vein for all patients except for 20 of the 29 patients who had a tunneled twin jugular venous catheter. Complications that occurred during ICU stay are summarized in Table 2.

Median length stays was 4 days ( $2-8 \mathrm{~d}$ ) in ICU and 10 days $(0-20 \mathrm{~d})$ in hospital (Table 2). Fifty-eight patients died in ICU $(41.4 \%)$ and seven in the hospital which brings the mortality at $46.4 \%$. In-hospital mortality was lower than that predicted by SAPS II score (Fig. S2, Supplemental Digital Content 3, http:// links.lww.com/CCM/F526; legend, Supplemental Digital Content 6, http://links.lww.com/CCM/F529). A control group of 140 ICU non-ESKD patients was matched by age, sex, SAPS II and SOFA scores, and ICU management to our ESKD population. ICU mortality was significantly lower in the control group (25\% vs 41.4\%; $p=0.005$ ) (Table S2, Supplemental Digital Content 4, http://links.lww.com/CCM/F527). Mortality after 90 days and 1 -year was at $50 \%$ and $62.9 \%$, respectively. Figure S3 (Supplemental Digital Content 5, http://links.lww. com/CCM/F528; legend, Supplemental Digital Content 6, http://links.lww.com/CCM/F529) represents the Kaplan-Meier curve for 1-year survival after admission to the ICU.

\section{Prognostic Analysis of the 140 CKD Patients Admitted to the ICU}

The univariate analysis of the data between ICU survivors and nonsurvivors is shown in Table 3. Among severity scores at ICU admission, nonrenal SOFA score greater than 8 was significantly associated with the mortality. Among biological variables studied upon ICU admission, the following were also significantly predictive of mortality: albuminemia less than $30 \mathrm{~g} / \mathrm{L}$, serum lactate greater than $4 \mathrm{mmol} / \mathrm{L}$, phosphatemia greater than $2.5 \mathrm{mmol} / \mathrm{L}$, serum bicarbonate less than 15 $\mathrm{mmol} / \mathrm{L}$. At ICU admission and during ICU course, the need for MV, for catecholamine, worsens significantly the prognosis. By multivariate analysis, nonrenal SOFA score greater than 8 , the need of invasive MV, and vasoconstrictive agents were the only variables independently related to the mortality (Table 3 ).

By univariate analysis, dialysis duration prior to admission greater than 3 years was significantly associated with 1-year mortality in addition to factors previously identified for ICU mortality (Table 4). However, multivariate analysis retained
TABLE 2. Clinical and Biological Variables at ICU Admission, Organs Support and Complications During ICU Stay, and Outcomes

Variables

$n=140$

ICU admission

SAPS $\|^{a}$, mean $\pm \mathrm{SD}$

$63 \pm 22$

SAPS II , median (IQR 25-75)

$61(45.5-81.25)$

Ventilatory support, $n$ (\%)

$100(71.4)$

Vasopressor drugs, $n$ (\%)

$90(64.3)$

SOFA ${ }^{a}$ excluding renal failure, mean $\pm \mathrm{SD}$

SOFA ${ }^{a}$ excluding renal failure, median (IOR 25-75)

Albuminemia <30 g/L, $n(\%)$

Albuminemia $(g / L)$, mean $\pm S D$ $6.7 \pm 3.8$

Albuminemia $^{a}(\mathrm{~g} / \mathrm{L})$, median (IOR 25-75)

Phosphatemia $<1.2 \mathrm{mmol} / \mathrm{L}, n(\%)$

Phosphatemia $>2.5 \mathrm{mmol} / \mathrm{L}, n(\%)$

Phosphatemia (mmol/L), median (IOR 25-75)

Lactate $\geq 4 \mathrm{mmol} / \mathrm{L}, n(\%)$

$7(3.75-10)$

88 (62.9)

$27.3 \pm 7.7$

27.5 (22-32.75)

$32(28.8)$

15 (13.5)

$1.55(1.15-2)$

48 (34.3)

ICU management

Ventilatory support, $n$ (\%)/duration, d $100(71.4) / 2(0-4.5)$ (IQR 25-75)

Vasopressor drugs, $n(\%) /$ duration, $d$ (IOR 25-75)

Blood transfusion, $n$ (\%)

$94(67.1) / 2(0-4)$

Complications during ICU stay, $n$ (\%)

Ventilator-acquired pneumonia

$24(20.9)$

Catheter-related infection

$10(7.1)$

Candidemia

Severe hemorrhage

$12(8.6)$

Outcomes

$\begin{array}{ll}\begin{array}{ll}\text { ICU length of stay, median (IOR } \\ \text { 25-75) }\end{array} & 4(2-8) \\ \text { In-ICU mortality, } n(\%) & 58(41.4) \\ \text { Limitation of care, } n(\%) & 42(30) \\ \begin{array}{l}\text { Hospital length of stay, median } \\ \quad \text { IOR 25-75) }\end{array} & 10(3.75-20.75) \\ \text { In-hospital mortality, } n(\%) & 65(46.4) \\ \text { Death at D90, } n(\%) & 70(50) \\ \text { Death at } 1 \mathrm{yr}, n(\%) & 88(62.9)\end{array}$

IOR = interquartile range, SAPS II = Simplified Acute Physiology Score, SOFA = Sequential Organ Failure Assessment.

aSAPS II, SOFA, and albuminemia were parametrically distributed. 


\section{TABLE 3. Predictive Factors of ICU Mortality of 140 Chronic Renal Dialysis Patients}

Admitted to the ICU

Univariate analysis

Age $>60 \mathrm{yr}$

$1.12(0.57-2.22)$

0.74

Male vs female

$1.03(0.6-1.8)$

Prior dialysis duration $>3 \mathrm{yr}$

$1.59(0.93-2.73)$

Charlson Comorbidity Index $>7$

$1.09(0.54-2.19)$

0.8

Albuminemia $<30 \mathrm{~g} / \mathrm{L}$

$2.22(1.14-4.32)$

Normal serum phosphorus

Reference

Phosphatemia $<1.2 \mathrm{mmol} / \mathrm{L}$

$0.7(0.32-1.49)$

Phosphatemia $>2.5 \mathrm{mmol} / \mathrm{L}$

$2.04(0.98-4.25)$

Serum lactate $>4 \mathrm{mmol} / \mathrm{L}$

Serum bicarbonate $<15 \mathrm{mmol} / L$

$3.05(1.78-5.25)$

Ventilatory support

$8.97(2.8-28.71)$

Norepinephrine $>0.1 \mu \mathrm{g} / \mathrm{kg} / \mathrm{min}$

$4.07(2.06-8.06)$

Nonrenal SOFA score $>8$

$4.89(2.81-8.52)$

$<0.001$

Readmission in ICU within $30 \mathrm{~d}$ after discharge

$0.43(0.06-3.13)$

Multivariate analysis

Ventilatory support

Norepinephrine $>0.1 \mu \mathrm{g} / \mathrm{kg} / \mathrm{min}$

$\mathrm{OR}=$ odds ratio, SOFA $=$ Sequential Organ Failure Assessment

Univariate and multivariate analysis.

only dialysis duration, nonrenal SOFA score greater than 8 , ICU admission albuminemia less than $30 \mathrm{~g} / \mathrm{L}$, and phosphatemia greater than $2.5 \mathrm{mmol} / \mathrm{L}$ as related risk factors of mortality (Table 4). Kaplan-Meier survival curves at 1 year according to previous duration of dialysis, nonrenal SOFA score, phosphatemia, and albuminemia at admission are shown in Figure 1.

\section{DISCUSSION}

The aim of this study was to reassess the epidemiology, the clinical features, and the outcome of critically ill CD patients. First, we observed that $1.7 \%$ of all patients admitted to the ICU over the examined 6-year period were receiving either chronic hemodialysis or peritoneal dialysis. We found that the mortality rate for this population was $41 \%$ in the ICU, and $46 \%$ when discharged from the hospital. More than two of three of the patients (52/76) discharged from the hospital survived 1 year later. Our results showed also that an increased scoring of organs dysfunction (SOFA $>8$ ), the need for either MV or catecholamines were the main indicators of ICU mortality. A previous dialysis duration greater than 3 years, "an ICU admission" albuminemia less than $30 \mathrm{~g} / \mathrm{L}$, and phosphoremia greater than $2.5 \mathrm{mmol} / \mathrm{L}$ were predictive of long-term mortality at 1 -vear in addition to SOFA score.
During the last decade, the incidence of chronic kidney disease has significantly increased mainly due to the aging population (1). Consequently, a large proportion of older ESKD patients which present several comorbidities are risky to severe organ dysfunction and admission to the ICU. Studies about critically ill ESKD patients reported an ICU mortality varying from $9 \%$ to $44 \%(9,10,15,16,24,25,30-33)$. In 2013, Arulkumaran et al (34) published a systematic review of these studies. He analyzed 16 studies, all performed before 2010, that included a total of 6,591 critically ill ESKD patients: two were prospective case-control studies, four were prospective cohort studies, and the remaining 10 were retrospective cohort studies. Mean patient ages were between 44 and 66 years (34), whereas that of our population was higher at 70 years (62-76 yr). Also, our patients, a 2012-2017 cohort, would have benefited from recent improvement of life expectancy of endstage renal diseases and from recent advances in life-sustaining therapies (35-37). Our first objective was therefore to compare preexisting data with the more recent data of our cohort in terms of requirement of ICU care, mortality, and risk factors of short- and long-term mortality.

Critically ill CRD patients were comparable to those of CRD povulation: similar mean age. and comorbidities (5). Among 


\section{TABLE 4. Predictive Factors of 1-Year Mortality of 140 Chronic Renal Dialysis Patients Admitted to the ICU}

Univariate analysis

\begin{tabular}{|c|c|c|}
\hline Age $>60 \mathrm{yr}$ & $1.07(0.63-1.82)$ & 0.79 \\
\hline Male vs female & $1.02(0.65-1.59)$ & 0.94 \\
\hline Prior dialysis duration $>3 \mathrm{yr}$ & $2.09(1.27-3.44)$ & 0.004 \\
\hline Charlson Comorbidity Index $>7$ & $1.03(0.59-1.80)$ & 0.92 \\
\hline Albuminemia $<30 \mathrm{~g} / \mathrm{L}$ & $2.33(1.4-3.91)$ & 0.001 \\
\hline Normal serum phosphorus & Reference & \\
\hline Phosphatemia $<1.2 \mathrm{mmol} / \mathrm{L}$ & $0.55(0.31-1.95)$ & 0.03 \\
\hline Phosphatemia $>2.5 \mathrm{mmol} / \mathrm{L}$ & $2.27(1.23-4.15)$ & 0.008 \\
\hline Serum lactate $>4 \mathrm{mmol} / \mathrm{L}$ & $2.18(1.42-3.34)$ & $<0.001$ \\
\hline Serum bicarbonate $<15 \mathrm{mmol} / \mathrm{L}$ & $1.94(1.20-3.14)$ & 0.007 \\
\hline Ventilatory support & $2.06(1.56-4.53)$ & $<0.001$ \\
\hline Norepinephrine $>0.1 \mu \mathrm{g} / \mathrm{kg} / \mathrm{min}$ & $2.86(1.78-4.6)$ & $<0.001$ \\
\hline Nonrenal SOFA score $>8$ & $3.54(2.3-5.45)$ & $<0.001$ \\
\hline Readmission in ICU within $30 \mathrm{~d}$ after discharge & $0.96(0.3-3.03)$ & 0.94 \\
\hline \multicolumn{3}{|l|}{ Multivariate analysis } \\
\hline Prior dialysis duration $>3 \mathrm{yr}$ & $1.57(1.08-2.48)$ & 0.048 \\
\hline Albuminemia $<30 \mathrm{~g} / \mathrm{L}$ & $2.05(1.2-3.51)$ & 0.01 \\
\hline Phosphatemia $>2.5 \mathrm{mmol} / \mathrm{L}$ & $2.21(1.17-4.15)$ & 0.02 \\
\hline Nonrenal SOFA score $>8$ & $2.8(1.76-4.45)$ & 0.001 \\
\hline
\end{tabular}

$\mathrm{OR}=$ odds ratio, SOFA $=$ Sequential Organ Failure Assessment.

Univariate and multivariate analysis.

all ICU admissions, they accounted for $1.7 \%$, a lower rate as compared to that previously reported (9). ICU admission rates as high as $8.6 \%$ have been reported (9) but with less severity as reflected by lower mean SAPS II (43 vs 63). Nonetheless, reasons of admission complied with those reported in the literature (34), sepsis mainly of pulmonary origin being the most often observed. The occurrence of sepsis may be facilitated by the specific underlying state of dysimmunity (38-40). ICU mortality rate in this population was at $41.4 \%$, higher than the observed overall mortality in our units (18-20\%). This rate was also higher than that previously reported $(20,34)$. Again, our patients were more severe and experienced mostly severe multiple organ failure (9). Chapman et al (32) found, in ESKD patients with multiple organ failure, a similar ICU mortality rate at $44 \%$. Although this mortality rate was correlated with SAPS II score, it was overestimated (9). We corroborated previous findings suggesting that SAPS II would not be a reliable severity score in ESKD patients $(34,41)$.

Long-term mortality was $50 \%$ at 3 months and $63 \%$ at 1 year after admission. Long-term outcome was worse than that of ESKD patients who did not experienced a severe illness requiring ICU admission. Indeed, 1-year life expectancy after RRT initiation is about $90 \%$ and $78 \%$ and 5-year life expectancy about $77 \%$ and $37 \%$ in patients older and younger than 65 years, respectively (7). Also, long-term outcome was obviously worse than that of ICU general population, but it would be better than that of others risky patients including acute renal failure treated by RRT or cirrhotic patients (11, 42-45). However, it should be underlined that more than two of three of discharged patients from the hospital survived at 1 year, which is a relatively favorable outcome. Furthermore, 11 of 26 patients who were admitted after a cardiac arrest were discharged from the hospital and eight of them were still alive 1 year later. Studies reporting survival at 1 year and beyond are limited $(15,30-32)$. Reported mortality rates varied from $35 \%$ to $60 \%$ with a trend for an increased mortality rate within the first 1-6 months after discharge and a relatively stable mortality thereafter, as we observed. Nonetheless, our results demonstrated meaningful short- and long-term survival and should therefore prompt intensivists not to consider ICU admission to be futile and not to avoid intensive therapy in critically ill CRD patients.

Our major concern in this study was the identification of predictive factors of both short- and long-term mortality. We 


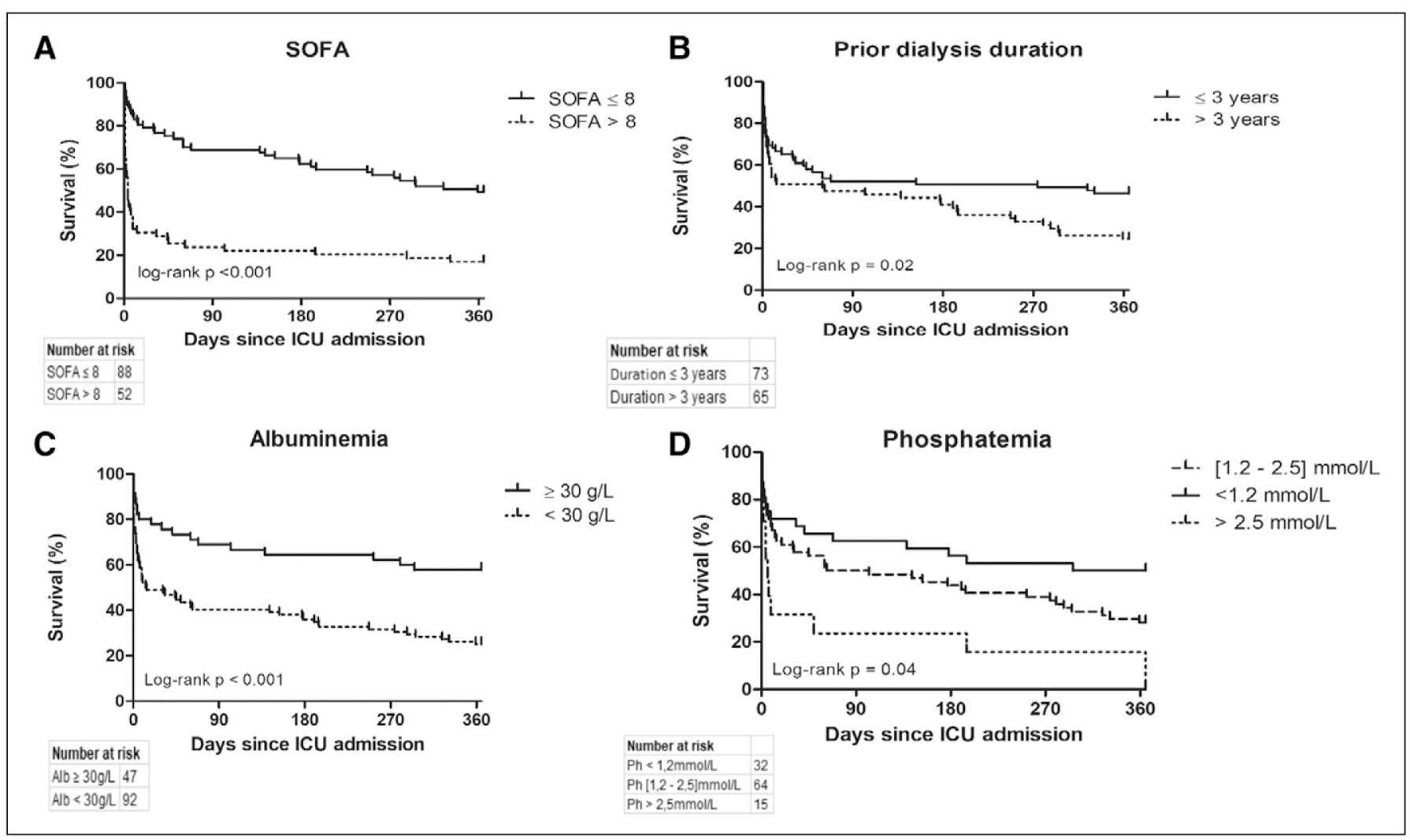

Figure 1. Kaplan-Meier survival curves at one year. One-yr survival of chronic renal disease patients after ICU admission according to: (A) Sequential Organ Failure Assessment (SOFA) score; (B) prior dialysis duration; (C) albuminemia (Alb); and (D) phosphatemia (Ph).

found that age and Charlson Comorbidity Index were not associated with ICU and hospital mortality. Charlson Comorbidity Index has been validated as a prognostic factor in elderly patient, incident patients on peritoneal dialysis and in oncology patients but not in chronic hemodialysis (46-49). By contrast, an admission lactatemia over $4 \mathrm{mmol} / \mathrm{L}$ and an increased phosphatemia were associated with ICU mortality $(9,45)$, but this association was weak since it was not confirmed by multivariate analysis. Multivariate analysis retained only SOFA score and the requirement of MV or/and vasopressor drugs as predictive factors of ICU mortality as demonstrated by other studies $(9-11,25,50)$. It strongly suggests that short-term mortality depends mainly on the number and severity of the acute organ failures associated to chronic renal failure $(9,16,26,51)$. It is noteworthy that SAPS II predicted mortality was greater than the actual mortality. By contrast, variables of nonrenal organ failure, namely nonrenal SOFA score, seems more useful. Data of SOFA score validity remains, however, conflicting with an area under the curve varying from 0.66 to $0.92(33,52)$ and require further validation. Yet, a significant number of patients died shortly after ICU discharge and within 90 days alerting physicians that ESKD patients may have a decreased physiologic reserve and are more susceptible to relapse from their original critical illness.

A dialysis duration over 3 years, an ICU admission albuminemia less than $30 \mathrm{~g} / \mathrm{L}$ and phosphatemia greater than $2.5 \mathrm{mmol} / \mathrm{L}$, and a nonrenal SOFA greater than 8 at admission predicted a worse long-term outcome at 1 year, whereas requirement of MV or/and catecholamines during ICU stay did not. Hyperphosphatemia may be an indicator of inadequate renal replacement (including medical therapy) and a risk factor for cardiovascular disease (9). Hypoalbuminemia is predictor of death in ESKD patients, yet its cause is multifactorial including malnutrition and an inflammatory state either acute or chronic $(53,54)$. It may also reflect the acute illness. Our results suggest that chronic treatment of ESKD may influence 1-year mortality by duration and quality of RRT as reflected by the level of phosphatemia and "probably" by patient's nutritional status (24). A score that take into account duration and quality of chronic dialysis, previous nutritional status and severity of organ involvement at ICU admission would best predict long-term outcome of critically ill ESKD patients and would help intensivists and nephrologists to best manage such a complication in accordance with family and patient wishes (55-59). It is noteworthy that, in our cohort, about 40 patients died as a result of a limitation or discontinuation of active therapies.

This study has several strengths. It was the most recent study on CRD patients admitted to the ICU. Additionally, consecutive inclusion of unselected critically ill ESKD patients may limit selection bias. Last, we were able to evaluate the longterm outcome. This study has also several limitations. This observational study was conducted in two ICUs in close contact with their Nephrology and Transplantation departments that may be not the case elsewhere. Furthermore, criteria for referral to ICU and ICU admission policies may vary. Indeed, 
patients that have not been admitted because of futile aggressive care were not included in our analysis. Consequently, our results should not be generalized to all ICUs. RRT related variables including fluid removal, pre- and intradialytic variables and hemodynamics, prior biological variables like anemia were not collected but they may alter outcome and should be further evaluated. Our follow-up lasted 1 year but it was demonstrated that critical illness would impact long-term mortality only the first 2 years and particularly the first 6 months. We must acknowledge that the occurrence of nonsevere event the year after hospital discharge has not been recorded; potential ICU readmission has been however collected. Last, we did not investigate the quality of life and associated morbidity after ICU discharge.

\section{CONCLUSIONS}

In summary, severe complications in ESKD patients are far from negligible, representing about $2 \%$ of all ICU admissions. They are mostly septic of pulmonary origin. Factors predictive of ICU and hospital mortality depend mainly on the severity of the critical event as reflected by nonrenal SOFA score and the need of MV and/or catecholamines. Long-term outcome is associated both with nutritional status and prior chronic dialysis treatment and with the number of organ failure at ICU admission. In order to improve the likelihood of survival in this population, further research is however still needed regarding critical care management and prediction of mortality. Also, there is a need for additional investigation to evaluate the impact of critical illness on readmissions, further risk of critical illness, quality of life, measures of frailty and functional status.

The authors have disclosed that they do not have any potential conflicts of interest.

For information regarding this article, E-mail: k-klouche@chu-montpellier.fr This study was performed in two medical ICUs of university hospitals in Montpellier and Nimes, France.

\section{REFERENCES}

1. Ruggenenti $P$, Schieppati $A$, Remuzzi G: Progression, remission, regression of chronic renal diseases. Lancet 2001; 357:1601-1608

2. Gilg J, Caskey F, Fogarty D: UK renal registry 18th annual report: Chapter 1 UK renal replacement therapy incidence in 2014: National and centre-specific analyses. Nephron 2016; 132(Suppl 1):9-40

3. Saran R, Robinson B, Abbott KC, et al: US renal data system 2016 annual data report: Epidemiology of kidney disease in the United States. Am J Kidney Dis 2017; 69:A7-A8

4. Pippias M, Stel VS, Abad Diez JM, et al: Renal replacement therapy in Europe: A summary of the 2012 ERA-EDTA Registry Annual Report. Clin Kidney J 2015; 8:248-261

5. The Renal Epidemiology and Information Network (REIN): French national end-stage renal disease registry: Annual Data Report 2016. Available at: https://www.agence-biomedecine.fr/lMG/pdf/rapportrein2016.pdf. Accessed May 19, 2019

6. Lassalle M, Monnet E, Ayav C, et al; REIN registry: 2017 annual report digest of the Renal Epidemiology Information Network (REIN) registry. Transp/ Int 2019; 32:892-902
7. The Renal Epidemiology and Information Network (REIN): French national end-stage renal disease registry: Annual Data Report 2017. Available at: https://www.agence-biomedecine.fr/IMG/pdf/rapportrein2017.pdf. Accessed May 19, 2020

8. Go AS, Chertow GM, Fan D, et al: Chronic kidney disease and the risks of death, cardiovascular events, and hospitalization. $N$ Engl J Med 2004; 351:1296-1305

9. Manhes G, Heng AE, Aublet-Cuvelier B, et al: Clinical features and outcome of chronic dialysis patients admitted to an intensive care unit. Nephrol Dial Transplant 2005; 20:1127-1133

10. Hutchison CA, Crowe AV, Stevens PE, et al: Case mix, outcome and activity for patients admitted to intensive care units requiring chronic renal dialysis: A secondary analysis of the ICNARC Case Mix Programme Database. Crit Care 2007; 11:R50

11. Rocha E, Soares M, Valente C, et al: Outcomes of critically ill patients with acute kidney injury and end-stage renal disease requiring rena replacement therapy: A case-control study. Nephrol Dial Transplant 2009: 24:1925-1930

12. Berthoux F, Jones E, Gellert R, et al: Epidemiological data of treated end-stage renal failure in the European Union (EU) during the year 1995: Report of the European Renal Association Registry and the National Registries. Nephrol Dial Transplant 1999; 14:2332-2342

13. Sarnak MJ, Jaber BL: Mortality caused by sepsis in patients with endstage renal disease compared with the general population. Kidney Int 2000; 58:1758-1764

14. De Rosa S, Samoni S, Villa G, et al: Management of chronic kidney disease patients in the intensive care unit: Mixing acute and chronic illness. Blood Purif 2017; 43:151-162

15. Strijack B, Mojica J, Sood M, et al: Outcomes of chronic dialysis patients admitted to the intensive care unit. J Am Soc Nephro/ 2009; 20:2441-2447

16. Clermont G, Acker CG, Angus DC, et al: Renal failure in the ICU: Comparison of the impact of acute renal failure and end-stage renal disease on ICU outcomes. Kidney Int 2002; 62:986-996

17. Uchino $S$, Morimatsu $H$, Bellomo $R$, et al: End-stage renal failure patients requiring renal replacement therapy in the intensive care unit: Incidence, clinical features, and outcome. Blood Purif 2003; 21:170175

18. Maizel J, Deransy R, Dehedin B, et al: Impact of non-dialysis chronic kidney disease on survival in patients with septic shock. BMC Nephrol 2013; 14:77

19. Wang HE, Gamboa C, Warnock DG, et al: Chronic kidney disease and risk of death from infection. Am J Nephrol 2011; 34:330-336

20. Chan M, Ostermann M: Outcomes of chronic hemodialysis patients in the intensive care unit. Crit Care Res Pract 2013; 2013:715807

21. Apel M, Maia VP, Zeidan M, et al: End-stage renal disease and outcome in a surgical intensive care unit. Crit Care 2013; 17:R298

22. Mansur A, Mulwande E, Steinau M, et al: Chronic kidney disease is associated with a higher 90-day mortality than other chronic medical conditions in patients with sepsis. Sci Rep 2015; 5:10539

23. Jung YS, Lee J, Shin HS, et al: Outcomes of patients with end-stage renal disease (ESRD) under chronic hemodialysis requiring continuous renal replacement therapy (CRRT) and patients without ESRD in acute kidney injury requiring CRRT: A single-center study. Hemodial Int 2012; 16:456-464

24. Sood MM, Miller L, Komenda P, et al: Long-term outcomes of endstage renal disease patients admitted to the ICU. Nephrol Dial Transplant 2011; 26:2965-2970

25. Ostermann M, Chang R; Riyadh ICU Program Users Group: Renal failure in the intensive care unit: Acute kidney injury compared to endstage renal failure. Crit Care 2008; 12:432

26. Le Gall JR, Lemeshow S, Saulnier F: A new Simplified Acute Physiology Score (SAPS II) based on a European/North American multicenter study. JAMA 1993; 270:2957-2963

27. Knaus WA, Wagner DP, Draper EA, et al: The APACHE III prognostic system. Risk prediction of hospital mortality for critically ill hospitalized adults. Chest 1991; 100:1619-1636

28. Charlson ME, Pompei P, Ales KL, et al: A new method of classifying prognostic comorbidity in longitudinal studies: Development and validation. J Chronic Dis 1987; 40:373-383 
29. Vincent JL, Moreno R, Takala J, et al: The SOFA (Sepsis-related Organ Failure Assessment) score to describe organ dysfunction/ failure. On behalf of the Working Group on Sepsis-Related Problems of the European Society of Intensive Care Medicine. Intensive Care Med 1996; 22:707-710

30. Bagshaw SM, Mortis G, Doig CJ, et al: One-year mortality in critically ill patients by severity of kidney dysfunction: A population-based assessment. Am J Kidney Dis 2006; 48:402-409

31. Bell M, Granath F, Schön S, et al; SWING: End-stage renal disease patients on renal replacement therapy in the intensive care unit: Short- and long-term outcome. Crit Care Med 2008; 36:2773-2778

32. Chapman RJ, Templeton M, Ashworth S, et al: Long-term survival of chronic dialysis patients following survival from an episode of multiple-organ failure. Crit Care 2009; 13:R65

33. Dara SI, Afessa B, Bajwa AA, et al: Outcome of patients with endstage renal disease admitted to the intensive care unit. Mayo Clin Proc 2004; 79:1385-1390

34. Arulkumaran N, Annear NM, Singer M: Patients with end-stage renal disease admitted to the intensive care unit: Systematic review. $\mathrm{Br} J$ Anaesth 2013; 110:13-20

35. Adhikari NK, Fowler RA, Bhagwanjee $S$, et al: Critical care and the global burden of critical illness in adults. Lancet 2010; 376:1339-1346

36. Foley RN, Collins AJ: End-stage renal disease in the United States: An update from the United States Renal Data System. J Am Soc Nephrol 2007; 18:2644-2648

37. Mokart D, Pastores SM, Darmon M: Has survival increased in cancer patients admitted to the ICU? Yes. Intensive Care Med 2014; 40:1570-1572

38. Peukert K, Wingender G, Patecki M, et al: Invariant natural killer T cells are depleted in renal impairment and recover after kidney transplantation. Nephrol Dial Transplant 2014; 29:1020-1028

39. Pop-Vicas A, Strom J, Stanley K, et al: Multidrug-resistant gram-negative bacteria among patients who require chronic hemodialysis. Clin J Am Soc Nephrol 2008; 3:752-758

40. Pesanti EL: Immunologic defects and vaccination in patients with chronic renal failure. Infect Dis Clin North Am 2001; 15:813-832

41. Klouche $\mathrm{K}$, Amigues $\mathrm{L}$, Massanet $\mathrm{P}$, et al: Outcome of renal transplant recipients admitted to an intensive care unit: A 10-year cohort study. Transplantation 2009; 87:889-895

42. O'Brien AJ, Welch CA, Singer M, et al: Prevalence and outcome of cirrhosis patients admitted to UK intensive care: A comparison against dialysis-dependent chronic renal failure patients. Intensive Care Med 2012; 38:991-1000

43. Thompson S, Pannu N: Dialysis patients and critical illness. Am J Kidney Dis 2012; 59:145-151

44. Rimes-Stigare $\mathrm{C}$, Frumento $\mathrm{P}$, Bottai $\mathrm{M}$, et al: Long-term mortality and risk factors for development of end-stage renal disease in critically ill patients with and without chronic kidney disease. Crit Care 2015; 19:383

45. Allegretti AS, Steele DJ, David-Kasdan JA, et al: Continuous renal replacement therapy outcomes in acute kidney injury and end-stage renal disease: A cohort study. Crit Care 2013; 17:R109

46. Buntinx F, Niclaes L, Suetens C, et al: Evaluation of Charlson's comorbidity index in elderly living in nursing homes. J Clin Epidemio/ 2002; 55:1144-1147

47. Fried L, Bernardini J, Piraino B: Charlson comorbidity index as a predictor of outcomes in incident peritoneal dialysis patients. $\mathrm{Am} J$ Kidney Dis 2001; 37:337-342

48. Lund L, Jacobsen J, Nørgaard M, et al: The prognostic impact of comorbidities on renal cancer, 1995 to 2006: A Danish population based study. J Urol 2009; 182:35-40; discussion 40

49. O'Connor KM, Davis N, Lennon GM, et al: Can we avoid surgery in elderly patients with renal masses by using the Charlson comorbidity index? BJU Int 2009; 103:1492-1495

50. Akbaş T, Karakurt S, Tuğlular S: Renal replacement therapy in the ICU: Comparison of clinical features and outcomes of patients with acute kidney injury and dialysis-dependent end-stage renal disease. Clin Exp Nephrol 2015; 19:701-709

51. Krinsley JS, Wasser T, Kang G, et al: Pre-admission functional status impacts the performance of the APACHE IV model of mortality prediction in critically ill patients. Crit Care 2017; 21:110

52. Juneja D, Prabhu MV, Gopal PB, et al: Outcome of patients with end stage renal disease admitted to an intensive care unit in India. Ren Fail 2010; 32:69-73

53. Kaysen GA, Rathore V, Shearer GC, et al: Mechanisms of hypoalbu minemia in hemodialysis patients. Kidney Int 1995; 48:510-516

54. Sarwar S, Sherman RA: How well does serum albumin correlate with dietary protein intake in dialysis patients? Kidney Int 2017; 2:89-93

55. Arulkumaran N, Szawarski P, Philips BJ: End-of-life care in patients with end-stage renal disease. Nephrol Dial Transplant 2012; 27:879881

56. Davison SN: Facilitating advance care planning for patients with endstage renal disease: The patient perspective. Clin J Am Soc Nephrol 2006; 1:1023-1028

57. Al-Jahdali $\mathrm{HH}$, Bahroon S, Babgi Y, et al: Advance care planning preferences among dialysis patients and factors influencing their decisions. Saudi J Kidney Dis Transp/ 2009; 20:232-239

58. Ostermann ME, Nelson SR: Haemodialysis patients' views on their resuscitation status. Nephrol Dial Transplant 2003; 18:16441647

59. Cohen LM, Germain MJ, Poppel DM: Practical considerations in dialysis withdrawal: "To have that option is a blessing." JAMA 2003; 289:2113-2119 